\title{
Multiplicity of symmetrically distinct sequences of solutions for a quasilinear problem in $\mathbb{R}^{N}$
}

\author{
Alexandru KRISTÁLY ${ }^{1}$ \\ University of Babeş-Bolyai \\ Department of Economics \\ 400591 Cluj-Napoca, Romania \\ e-mail: alexandrukristaly@yahoo.com \\ Waclaw MARZANTOWICZ ${ }^{2}$ \\ Adam Mickiewicz University \\ Faculty of Mathematics and Computer Science \\ 61-614 Poznań, Poland \\ e-mail: marzan@amu.edu.pl
}

\begin{abstract}
The present paper is concerned with an elliptic problem in $\mathbb{R}^{N}$ which involves the $p$-Laplacian, $p>N,(N=4$ or $N \geq 6)$, while the nonlinear term has an oscillatory behaviour and is odd near an arbitrarily small neighborhood of the origin. A direct variational argument and a careful group-theoretical construction show the existence of at least $\left[\frac{N-3}{2}\right]+(-1)^{N}$ sequences of arbitrary small, non-radial, sign-changing solutions such that elements in different sequences are distinguished by their symmetry properties.
\end{abstract}

2000 Mathematics Subject Classification: 35J20, 35J60, 35J70.

Key words: $p$-Laplacian; oscillatory nonlinearity; symmetrically distinct soltuions.

\section{Introduction}

The purpose of this paper is to guarantee by a direct variational method the existence of infinitely many non-radial and sign-changing solutions of the problem

$$
\left\{\begin{array}{l}
-\triangle_{p} u+|u|^{p-2} u=K(x) f(u), \quad x \in \mathbb{R}^{N}, \\
u \in W^{1, p}\left(\mathbb{R}^{N}\right),
\end{array}\right.
$$

\footnotetext{
${ }^{1}$ The research of A. Kristály was supported by the CNCSIS, project no. AT 8/70.

${ }^{2}$ The research of W. Marzantowicz was supported by KBN grant 1PO3A 03929.
} 
when $p>N$, the space dimension $N$ is large enough, and $f$ has an oscillatory behaviour at the origin. Here, $\triangle_{p} u=\operatorname{div}\left(|\nabla u|^{p-2} \nabla u\right)$ is the usual $p$-Laplacian of $u, K: \mathbb{R}^{N} \rightarrow \mathbb{R}$ is a measurable function, and $f: \mathbb{R} \rightarrow \mathbb{R}$ is continuous.

This study can be fit within a very active research area of the PDEs, i.e., to find sign-changing (nodal) solutions of various elliptic equations. We refer the reader to [1]-[5], [15], [22], and references therein for results of this type. Most of the papers treat semilinear problems due to the ordered Hilbert space structure of the associated functional space to the studied problem, see [1], [3], [15]. To the best of our knowledge, only a few works are dealing with sign-changing solutions of quasilinear problems (but only on bounded domains), see [2], [22]. In the aforementioned papers, the nonlinearities have superlinear and subcritical growth at infinity; the strategy is to construct suitable closed convex sets containing all the positive and negative solutions which are invariant with respect to some pseudo-gradient vector fields.

As we pointed out, our aim is to produce by a direct method many sign-changing and non-radial solutions for problem $(\mathrm{P})$ when $p>N$, and the nonlinearity $f$ has an oscillatory behaviour at the origin. Note that within this framework, problem (P) has been studied recently in [11] and [12]. As an effect of the oscillatory behavior of $f$ (with no additional assumption on the symmetry of $f$ ), in [11], [12] the authors obtained infinitely many non-negative solutions which are $G$-invariant (perhaps, radial symmetric), where $G$ are special subgroups of the orthogonal group $O(N)$. However, when we are interested in the existence of infinitely many non-radial, sign-changing solutions of $(\mathrm{P})$, it seems some kind of symmetry hypothesis on the nonlinearity $f$ is indispensable. In order to fill this gap, we require that $f$ is odd in an arbitrarily small neighborhood of the origin. A similar hypothesis has been used first by Wang [21] who studied a boundary value problem involving a concave nonlinearity near the origin, adapting Lusternik-Schnirelmann theory.

The weak solutions of $(\mathrm{P})$ are precisely the critical points of the energy functional $E: W^{1, p}\left(\mathbb{R}^{N}\right) \rightarrow \mathbb{R}$ associated to problem $(\mathrm{P})$ which is defined by

$$
E(u)=\frac{1}{p} \int_{\mathbb{R}^{N}}\left(-\triangle_{p} u+|u|^{p-2} u\right) u-\int_{\mathbb{R}^{N}} K(x) F(u(x)) d x .
$$

Here, $F(s)=\int_{0}^{s} f(t) d t, s \in \mathbb{R}$. In order to produce a sequence of non-radial, sign-changing weak solutions of $(\mathrm{P})$ we first introduce a new energy functional $\bar{E}$ which is 'close' to $E$ and is even, exploiting the fact that $f$ is odd near the origin. Then, we restrict $\bar{E}$ to a carefully chosen subspace $Z$ of $W^{1, p}\left(\mathbb{R}^{N}\right)$. Here, the space $Z$ consists of functions with special symmetrical properties. As a consequence of this symmetry property, $Z$ contains only sign-changing and non-radial elements of $W^{1, p}\left(\mathbb{R}^{N}\right)$. Next, we construct an appropriate sequence of subsets of $Z$. The main step is to show that the relative minima of $\bar{E}_{\mid Z}$ in these subsets of $Z$ are local minima of $\bar{E}_{\mid Z}$. A careful analysis shows that one can extract infinitely many local minima of $\bar{E}_{\mid Z}$ with arbitrarily small $L^{\infty}$-norms. Moreover, these local minima of $\bar{E}_{\mid Z}$ are critical points of $\bar{E}$, due to an invariance property of $\bar{E}$ and to the principle 
of symmetric criticality. In particular, on account of the construction of $\bar{E}$, the elements belonging to this sequence are actually distinct weak solutions of $(\mathrm{P})$.

This fact leads us to the following natural question: how many sequences of non-radial, sign-changing weak solutions of $(\mathrm{P})$ can we produce at least whose elements are mutually symmetrically different? Note that Bartsch and Willem [5] handled a related question for $(\mathrm{P})$ in the semilinear case (i.e., $p=2$ ) when $N=4$ or $N \geq 6$. By means of the Fountain Theorem, they obtained a sequence $\left\{u_{k}^{l}\right\}_{k \in \mathbb{N}}$ of non-radial, sign-changing solutions of the studied problem for every $l \in I_{N}:=$ $\{i \in \mathbb{N}: 2 \leq i \leq N / 2,2 i \neq N-1\}$. Bartsch and Willem asked whether the solutions $u_{k}^{l}$ and $u_{n}^{m}$ lie on the same $O(N)$-orbit when $l \neq m$. They obtained the following answer: for every $N \geq 4, N \neq 5$, the number of those sequences of solutions containing elements in different $O(N)$-orbits is at least $\left[\log _{2} \frac{N+2}{3}\right]$, as a careful inspection of $[5$, Proposition 4.1, p. 457] shows. (Here, $[r]$ denotes the integer part of $r$.) Coming back to our question, one could expect that the number we are looking for increases with respect to the space dimension $N$. However, we are able to show that the number of sequences of non-radial, sign-changing weak solutions of $(\mathrm{P})$ - such that elements in different sequences are mutually distinguished by their symmetry properties - is at least $s_{N}=$ card $I_{N}=\left[\frac{N-3}{2}\right]+(-1)^{N}$. Note that $s_{N} \sim N / 2$ as $N \rightarrow \infty$, but the sequence $\left\{s_{N}\right\}_{N \geq 4}$ is not increasing, and $s_{5}=0$. The reason is that the number $s_{N}$ does not depend only on the space dimension $N$ but also on special partitions of $N$. The fact that $s_{5}=0$ shows unfortunately that our argument to produce non-radial, sign-changing solutions for $(\mathrm{P})$ fails whenever $N=5$; a same phenomenon has been pointed out in [4] and [5] as well.

The paper is organized as follows. In Section 2 we will formulate our main result. In Section 3 we prove the existence of a sequence of weak solutions of (P) for a suitably chosen subspace of $W^{1, p}\left(\mathbb{R}^{N}\right)$ which are sign-changing nonradial by the symmetry property of the subspace. In the last section, we construct $s_{N}$ special subspaces of $W^{1, p}\left(\mathbb{R}^{N}\right)$ for which one can apply the arguments from Section 3 and these subspaces have only the 0 as a common element. In this way, we produce $s_{N}$ sequences of solutions of $(\mathrm{P})$ which do no contain similar elements from symmetrical point of view.

\section{Statement of the main result}

First of all, we recall that the space $W^{1, p}\left(\mathbb{R}^{N}\right)$ is endowed with the norm $\|u\|_{W^{1, p}}=$ $\left(\|\nabla u\|_{p}^{p}+\|u\|_{p}^{p}\right)^{1 / p}$ where $\|\cdot\|_{p}$ is the usual norm in $L^{p}\left(\mathbb{R}^{N}\right), 1<p<\infty$. The space $L^{\infty}\left(\mathbb{R}^{N}\right)$ is endowed with the sup-norm, denoted by $\|\cdot\|_{\infty}$.

In this section we will give the precise form of our main result. Throughout the paper we assume that the potential $K: \mathbb{R}^{N} \rightarrow \mathbb{R}$ fulfills the hypothesis:

$\left(K_{1}\right) K \in L^{1}\left(\mathbb{R}^{N}\right) \cap L^{\infty}\left(\mathbb{R}^{N}\right)$ is radial, non-negative, and $\|K\|_{\infty}>0$.

The nonlinearity $f: \mathbb{R} \rightarrow \mathbb{R}$ exhibits an oscillatory behaviour at the origin in the following sense: 
$\left(f_{1}\right)-\infty<\liminf _{s \rightarrow 0} \frac{F(s)}{|s|^{p}} \leq \limsup _{s \rightarrow 0} \frac{F(s)}{|s|^{p}}=+\infty$; here, $F(s)=\int_{0}^{s} f(t) d t$.

$\left(f_{2}\right)$ There are two sequences $\left\{a_{k}\right\}_{k \in \mathbb{N}},\left\{b_{k}\right\}_{k \in \mathbb{N}}$ such that $0<b_{k+1}<a_{k}<b_{k}$, $\lim _{k \rightarrow \infty} b_{k}=0$, and $f(s) \leq 0$ for every $s \in\left[a_{k}, b_{k}\right], k \in \mathbb{N}$.

Our main result can be stated as follows:

Theorem 2.1 Let $p>N$ and $N=4$ or $N \geq 6$. Let $K: \mathbb{R}^{N} \rightarrow \mathbb{R}$ be a function which satisfies $\left(K_{1}\right)$, and $f: \mathbb{R} \rightarrow \mathbb{R}$ be a continuous function which is odd in a neighborhood of the origin and verifies $\left(f_{1}\right)$ and $\left(f_{2}\right)$, respectively.

Then there exist at least $s_{N}=\left[\frac{N-3}{2}\right]+(-1)^{N}$ sequences $\left\{u_{k}^{l}\right\}_{k \in \mathbb{N}} \subset$ $W^{1, p}\left(\mathbb{R}^{N}\right), l \in\left\{1, \ldots, s_{N}\right\}$, of homoclinic, non-radial, sign-changing weak solutions of $(\mathrm{P})$ such that elements in different sequences are distinguished by their symmetry properties. In addition, $\lim _{k \rightarrow \infty}\left\|u_{k}^{l}\right\|_{\infty}=0$ for every $l \in\left\{1, \ldots, s_{N}\right\}$.

Remark 2.1 Different sequences of solutions for $(\mathrm{P})$ will be constructed in carefully chosen subspaces of $W^{1, p}\left(\mathbb{R}^{N}\right)$; the difference between them is emphasized by the symmetrical properties of functions belonging to the subspaces (see Section 4). These subspaces are obtained via a group-theoretical argument carried out on certain subgroups of the orthogonal group $O(N)$. Note that the unique common element of these subspaces is 0; consequently, elements of different sequences from these subspaces of $W^{1, p}\left(\mathbb{R}^{N}\right)$ cannot be compared from symmetrical point of view.

Remark 2.2 Problem (P) has been widely studied when $N \geq p$. In the semilinear case (i.e., $N \geq p=2$ ), certain solitary waves in the nonlinear Klein-Gordon and Schödinger equations are solutions of $(\mathrm{P})$; existence and multiplicity of solutions can be found for instance in [3], [5], and references therein. In the quasilinear case (i.e., $N \geq p \neq 2$ ), problem (P) was treated in [9], [14]. The common feature of these papers is the superlinear and subcritical growth at infinity of the nonlinear term $f$. Theorem 2.1 complements several results mentioned above; we treat the case $p>N$ which is also important from the Mathematical Physics point of view (see [6], [10]), while the nonlinear term $f$ is allowed to have an unusual behavior. Note that closely related hypotheses to $\left(f_{1}\right)$ and $\left(f_{2}\right)$ have been used in [19], [20] studying boundary value problems.

Example 2.1 Let $p>3, q \in(p-3, p-2)$ and $\delta>0$ be fixed arbitrarily. A nonlinearity $f: \mathbb{R} \rightarrow \mathbb{R}$ verifying $\left(f_{1}\right)$ and $\left(f_{2}\right)$ is

$$
f(s)=\left\{\begin{array}{lll}
|s|^{q} \sin \frac{1}{s}, & \text { if } & s \in[-\delta, \delta] \backslash\{0\} \\
0, & \text { if } & s=0 \\
g(s), & \text { if } & |s|>\delta
\end{array}\right.
$$

where $g: \mathbb{R} \backslash[-\delta, \delta] \rightarrow \mathbb{R}$ is any continuous function with $g(\delta)=-g(-\delta)=\delta^{q} \sin \frac{1}{\delta}$. 
Remark 2.3 In problem (P), instead of the operator $u \mapsto-\triangle_{p} u+|u|^{p-2} u$ we may put $u \mapsto-\triangle_{p} u+V(x)|u|^{p-2} u$ where $V: \mathbb{R}^{N} \rightarrow \mathbb{R}$ is radial and is bounded from below by a positive constant.

\section{Existence of a sequence of non-radial, sign-changing solutions for $(\mathrm{P})$}

Throughout of this section, we assume that the assumptions of Theorem 2.1 are fulfilled. The energy functional $E: W^{1, p}\left(\mathbb{R}^{N}\right) \rightarrow \mathbb{R}$ associated to problem (P)

$$
E(u)=\frac{1}{p}\|u\|_{W^{1, p}}^{p}-\int_{\mathbb{R}^{N}} K(x) F(u(x)) d x, \quad u \in W^{1, p}\left(\mathbb{R}^{N}\right),
$$

is well-defined, where $F(s)=\int_{0}^{s} f(t) d t, s \in \mathbb{R}$. Indeed, since $p>N$, Morrey's embedding theorem implies that $W^{1, p}\left(\mathbb{R}^{N}\right)$ is continuously embedded into $L^{\infty}\left(\mathbb{R}^{N}\right)$. Therefore, due to $\left(K_{1}\right)$, the second term of (1) is finite for every $u \in W^{1, p}\left(\mathbb{R}^{N}\right)$. Moreover, one can show in a standard way that $E$ is of class $C^{1}$ on $W^{1, p}\left(\mathbb{R}^{N}\right)$ and its critical points are exactly the weak solutions of $(\mathrm{P})$, see [13]. It is well-known that one can consider continuous representations of the elements from $W^{1, p}\left(\mathbb{R}^{N}\right)$, and every element $u$ from $W^{1, p}\left(\mathbb{R}^{N}\right)$ is homoclinic, see [8, p. 167], i.e., $u(x) \rightarrow 0$ as $|x| \rightarrow \infty$.

Let $\delta>0$ such that $f_{[[-\delta, \delta]}$ is odd. Without to loose the generality, we may assume that $b_{1} \leq \delta$, i.e., the sequence $\left\{b_{k}\right\}_{k \in \mathbb{N}}$ appearing in $\left(f_{2}\right)$ does belong to $[0, \delta]$. Let $\bar{f}: \mathbb{R} \rightarrow \mathbb{R}$ be defined by $\bar{f}(t)=f(t)$ for $|t| \leq \delta$, and $\bar{f}(t)=\operatorname{sign}(t) f(\delta)$ for $|t|>\delta$. Put $\bar{F}(s)=\int_{0}^{s} \bar{f}(t) d t$. Let $\bar{E}: W^{1, p}\left(\mathbb{R}^{N}\right) \rightarrow \mathbb{R}$ be the new energy functional associated to problem $(\mathrm{P})$

$$
\bar{E}(u)=\frac{1}{p}\|u\|_{W^{1, p}}^{p}-\int_{\mathbb{R}^{N}} K(x) \bar{F}(u(x)) d x, \quad u \in W^{1, p}\left(\mathbb{R}^{N}\right) .
$$

It is clear that $\bar{E}$ is well-defined, is of class $C^{1}$ and is even.

Let $N \geq 4$. Define the sets

$$
\begin{aligned}
P_{N}=\{G \subset O(N): & G=O\left(N_{1}\right) \times \cdots \times O\left(N_{k}\right), k \geq 2, \\
& \left.N_{1}+\cdots+N_{k}=N, N_{j} \geq 2, j=1, \ldots, k\right\},
\end{aligned}
$$

and

$$
\tilde{P}_{N}=\left\{G=O\left(N_{1}\right) \times \cdots \times O\left(N_{k}\right) \in P_{N}: \exists i, j \in\{1, \ldots, k\}, i \neq j, N_{i}=N_{j}\right\} .
$$

Note that $\tilde{P}_{5}=\emptyset$. For every $G=O\left(N_{1}\right) \times \cdots \times O\left(N_{k}\right) \in \tilde{P}_{N}(N \neq 5)$ we define the set

$$
\begin{aligned}
N(G)=\{\tau \in O(N): & \tau\left(x_{1}, \ldots, x_{i}, \ldots, x_{j}, \ldots, x_{k}\right)=\left(x_{1}, \ldots, x_{j}, \ldots, x_{i}, \ldots, x_{k}\right), \\
& \left.\left(x_{1}, \ldots, x_{k}\right) \in \mathbb{R}^{N_{1}} \times \ldots \times \mathbb{R}^{N_{k}}, N_{i}=N_{j}, i \neq j\right\} .
\end{aligned}
$$


One can observe that for every $\tau \in N(G)$ we have $\tau \notin G, \tau G \tau^{-1}=G$ and $\tau=\tau^{-1}$; in particular, $N(G)$ is a subset of the normalizer of $G$ in $O(N)$.

For every fixed $G \in \tilde{P}_{N}$ and $\tau \in N(G)$ we introduce an action of the group $G_{\tau}=\langle G, \tau\rangle$ on the space $W^{1, p}\left(\mathbb{R}^{N}\right)$, as in [4], [5]. Due to the above properties of $\tau$, only two types of elements in $G_{\tau}$ can be distinguished; namely, $g \in G$, and $\tau g \in G_{\tau} \backslash G$ (with $g \in G$ ), respectively. Therefore, the action $G_{\tau} \times W^{1, p}\left(\mathbb{R}^{N}\right) \rightarrow$ $W^{1, p}\left(\mathbb{R}^{N}\right)$ given by

$$
g u(x)=u\left(g^{-1} x\right), \quad(\tau g) u(x)=-u\left(g^{-1} \tau^{-1} x\right),
$$

for every $g \in G, u \in W^{1, p}\left(\mathbb{R}^{N}\right)$ and $x \in \mathbb{R}^{N}$, is well-defined. Finally, for every $G \in \tilde{P}_{N}$ and $\tau \in N(G)$ we define the space

$$
W_{G_{\tau}}^{1, p}\left(\mathbb{R}^{N}\right)=\left\{u \in W^{1, p}\left(\mathbb{R}^{N}\right): h u=u \text { for every } h \in G_{\tau}\right\}
$$

which is nothing but the fixed point space of $W^{1, p}\left(\mathbb{R}^{N}\right)$ under the action $G_{\tau}$.

Remark 3.1 Note that every nonzero element of the space $W_{G_{\tau}}^{1, p}\left(\mathbb{R}^{N}\right)$ changes sign and is non-radial. To see this, let $u \in W_{G_{\tau}}^{1, p}\left(\mathbb{R}^{N}\right) \backslash\{0\}$. The first fact follows from the $G_{\tau}$-invariance of $u$ and (2). Indeed, in particular,

$$
u(x)=\tau u(x)=-u\left(\tau^{-1} x\right)
$$

for every $x \in \mathbb{R}^{N}$. Since $u \neq 0$, it should change the sign. Now, suppose that $u$ is radial. Since $|x|=\left|\tau^{-1} x\right|$, from (3) we obtain $u(x)=u(|x|)=0$, contradiction.

In the rest of this section, we will fix a $G \in \tilde{P}_{N}$ and $\tau \in N(G)$. The restriction of $\bar{E}$ to the space $W_{G_{\tau}}^{1, p}\left(\mathbb{R}^{N}\right)$ will be denoted by $\bar{E}_{\tau}$. Now, we define a sequence of subsets of $W_{G_{\tau}}^{1, p}\left(\mathbb{R}^{N}\right)$ by

$$
S_{k}=\left\{u \in W_{G_{\tau}}^{1, p}\left(\mathbb{R}^{N}\right):\|u\|_{\infty} \leq b_{k}\right\},
$$

where the sequence $\left\{b_{k}\right\}$ appears in the hypothesis $\left(f_{2}\right)$.

Lemma 3.1 $\bar{E}_{\tau}$ is bounded from below on $S_{k}$ and it attains its infimum on $S_{k}$.

Proof. We first prove that $\bar{E}_{\tau}$ is sequentially weakly lower semicontinuous on $W_{G_{\tau}}^{1, p}\left(\mathbb{R}^{N}\right)$. It is a standard fact that the norm-function $u \mapsto \frac{1}{p}\|u\|_{W^{1, p}}^{p}, u \in$ $W_{G_{\tau}}^{1, p}\left(\mathbb{R}^{N}\right)$ is sequentially weakly lower semicontinuous on $W_{G_{\tau}}^{1, p}\left(\mathbb{R}^{N}\right)$. It is sufficient to prove that the function $u \rightarrow \mathcal{F}_{K}(u)=\int_{\mathbb{R}^{N}} K(x) \bar{F}(u(x)) d x$ is sequentially weakly continuous on $W_{G_{\tau}}^{1, p}\left(\mathbb{R}^{N}\right)$. By contradiction, let $\left\{u_{n}\right\} \subset W_{G_{\tau}}^{1, p}\left(\mathbb{R}^{N}\right)$ be a sequence which converges weakly to $u \in W_{G_{\tau}}^{1, p}\left(\mathbb{R}^{N}\right)$ but $\mathcal{F}_{K}\left(u_{n}\right) \nrightarrow \mathcal{F}_{K}(u)$ as $n \rightarrow \infty$. Up to a subsequence, we can fix a number $\varepsilon_{0}>0$ such that

$$
0<\varepsilon_{0} \leq\left|\mathcal{F}_{K}\left(u_{n}\right)-\mathcal{F}_{K}(u)\right| \text { for every } n \in \mathbb{N} \text {. }
$$


Moreover, due to a result of Lions (see [16, Théorème III.3]), the embedding $W_{G}^{1, p}\left(\mathbb{R}^{N}\right) \hookrightarrow L^{q}\left(\mathbb{R}^{N}\right)$ is compact for every $q \in(p, \infty)$. In particular, the embedding $W_{G_{\tau}}^{1, p}\left(\mathbb{R}^{N}\right) \hookrightarrow L^{q}\left(\mathbb{R}^{N}\right)$ is also compact. Thus, fixing some $q \in(p, \infty)$ one may assume that $u_{n}$ converges strongly to $u$ in $L^{q}\left(\mathbb{R}^{N}\right)$. For every $n \in \mathbb{N}$ one has $\theta_{n} \in(0,1)$ such that

$$
\begin{aligned}
0 & <\varepsilon_{0} \leq\left|\mathcal{F}_{K}\left(u_{n}\right)-\mathcal{F}_{K}(u)\right| \leq \int_{\mathbb{R}^{N}} K(x)\left|\bar{f}\left(u+\theta_{n}\left(u_{n}-u\right)\right)\right| \cdot\left|u_{n}-u\right| d x \\
& \leq\|K\|_{q^{\prime}} \max \left\{|\bar{f}(s)|: 0 \leq|s| \leq M_{n}\right\}\left\|u_{n}-u\right\|_{q},
\end{aligned}
$$

where $q^{\prime}=q(q-1)^{-1}$ and $M_{n}=\|u\|_{\infty}+\left\|u_{n}\right\|_{\infty}$. Since $W_{G_{\tau}}^{1, p}\left(\mathbb{R}^{N}\right) \subset W^{1, p}\left(\mathbb{R}^{N}\right)$ is continuously embedded into $L^{\infty}\left(\mathbb{R}^{N}\right)$, we have $\sup _{n \in \mathbb{N}} M_{n}<+\infty$. When $n \rightarrow \infty$, the above inequality yields a contradiction.

One can observe that $S_{k}$ is weakly closed. Indeed, $S_{k}$ is convex and is strongly closed in $W_{G_{\tau}}^{1, p}\left(\mathbb{R}^{N}\right)$ since the embedding $W_{G_{\tau}}^{1, p}\left(\mathbb{R}^{N}\right) \subset W^{1, p}\left(\mathbb{R}^{N}\right) \hookrightarrow L^{\infty}\left(\mathbb{R}^{N}\right)$ is continuous. Moreover, for every $u \in S_{k}$ one has

$$
\bar{E}_{\tau}(u)=\frac{1}{p}\|u\|_{W^{1, p}}^{p}-\int_{\mathbb{R}^{N}} K(x) \bar{F}(u(x)) d x \geq-\|K\|_{1} \max _{\left[-b_{k}, b_{k}\right]} \bar{F} .
$$

Therefore, $\bar{E}_{\tau}$ is bounded from below on $S_{k}$. Let $\gamma_{k}=\inf _{S_{k}} \bar{E}_{\tau}$, and $\left\{u_{n}\right\}$ be a minimizing sequence of $\bar{E}_{\tau}$ in $S_{k}$ for $\gamma_{k}$. Then,

$$
\frac{1}{p}\left\|u_{n}\right\|_{W^{1, p}}^{p} \leq \gamma_{k}+1+\|K\|_{1} \max _{\left[-b_{k}, b_{k}\right]} \bar{F}
$$

for large $n \in \mathbb{N}$. Thus, $\left\{u_{n}\right\}$ is bounded in $W_{G_{\tau}}^{1, p}\left(\mathbb{R}^{N}\right)$. So, up to a subsequence, $\left\{u_{n}\right\}$ weakly converges in $W_{G_{\tau}}^{1, p}\left(\mathbb{R}^{N}\right)$ to some $\bar{u}_{k} \in S_{k}$. By the sequentially weakly lower semicontinuity of $\bar{E}_{\tau}$ we conclude $\bar{E}_{\tau}\left(\bar{u}_{k}\right)=\gamma_{k}=\inf _{S_{k}} \bar{E}_{\tau}$.

Proposition 3.1 Let $\bar{u}_{k} \in S_{k}$ be such that $\bar{E}_{\tau}\left(\bar{u}_{k}\right)=\inf _{S_{k}} \bar{E}_{\tau}$. Then, $\left\|\bar{u}_{k}\right\|_{\infty} \leq a_{k}$.

Proof. Let $A=\left\{x \in \mathbb{R}^{N}: \bar{u}_{k}(x) \notin\left[-a_{k}, a_{k}\right]\right\}$ and suppose that $A \neq \emptyset$. Thus, $\operatorname{meas}(A)>0$ due to the continuity of $\bar{u}_{k}$. Define the function $\phi: \mathbb{R} \rightarrow \mathbb{R}$ by $\phi(s)=\operatorname{sgn}(s) \min \left\{|s|, a_{k}\right\}$ and set $w_{k}=\phi \circ \bar{u}_{k}$. Since $\phi$ is uniformly Lipschitz and $\phi(0)=0$, the function $w_{k}$ belongs to $W^{1, p}\left(\mathbb{R}^{N}\right)$, cf. Marcus-Mizel [17]. Moreover, we claim that $w_{k} \in W_{G_{\tau}}^{1, p}\left(\mathbb{R}^{N}\right)$. To see this, it suffices to prove that $h w_{k}=w_{k}$ for every $h \in G_{\tau}$. First, let $h=g \in G$. Since $g \bar{u}_{k}=\bar{u}_{k}$, one has $g w_{k}(x)=w_{k}\left(g^{-1} x\right)=$ $\left(\phi \circ \bar{u}_{k}\right)\left(g^{-1} x\right)=\phi\left(\bar{u}_{k}\left(g^{-1} x\right)\right)=\phi\left(\bar{u}_{k}(x)\right)=w_{k}(x)$ for every $x \in \mathbb{R}^{N}$. Now, let $h=\tau g \in G_{\tau} \backslash G, g \in G$. Since $\phi$ is an odd function and $(\tau g) \bar{u}_{k}=\bar{u}_{k}$, on account of (2) we have

$$
\begin{aligned}
(\tau g) w_{k}(x) & =-w_{k}\left(g^{-1} \tau^{-1} x\right)=-\left(\phi \circ \bar{u}_{k}\right)\left(g^{-1} \tau^{-1} x\right) \\
& =\phi\left(-\bar{u}_{k}\left(g^{-1} \tau^{-1} x\right)\right)=\phi\left((\tau g) \bar{u}_{k}(x)\right)=\phi\left(\bar{u}_{k}(x)\right) \\
& =w_{k}(x)
\end{aligned}
$$


for every $x \in \mathbb{R}^{N}$. In conclusion, $w_{k} \in W_{G_{\tau}}^{1, p}\left(\mathbb{R}^{N}\right)$. By construction, $\left|w_{k}(x)\right| \leq$ $a_{k}<b_{k}$ for every $x \in \mathbb{R}^{N}$. Thus, $w_{k} \in S_{k}$.

Let us introduce the sets

$$
A^{-}=\left\{x \in A: \bar{u}_{k}(x)<-a_{k}\right\} \quad \text { and } \quad A^{+}=\left\{x \in A: \bar{u}_{k}(x)>a_{k}\right\} .
$$

Then, we have

$$
w_{k}(x)=\left\{\begin{array}{cc}
\bar{u}_{k}(x), & x \in \mathbb{R}^{N} \backslash A ; \\
-a_{k}, & x \in A^{-} \\
a_{k}, & x \in A^{+} .
\end{array}\right.
$$

A straightforward calculation shows that

$$
\begin{aligned}
\bar{E}_{\tau}\left(w_{k}\right)-\bar{E}_{\tau}\left(\bar{u}_{k}\right)= & -\frac{1}{p} \int_{A}\left|\nabla \bar{u}_{k}\right|^{p}+\frac{1}{p} \int_{A}\left[\left|w_{k}\right|^{p}-\left|\bar{u}_{k}\right|^{p}\right] \\
& -\int_{A} K(x)\left[\bar{F}\left(w_{k}\right)-\bar{F}\left(\bar{u}_{k}\right)\right] \\
= & -\frac{1}{p} \int_{A}\left|\nabla \bar{u}_{k}\right|^{p}+\frac{1}{p} \int_{A^{-}}\left[a_{k}^{p}-\left|\bar{u}_{k}\right|^{p}\right]+\frac{1}{p} \int_{A^{+}}\left[a_{k}^{p}-\bar{u}_{k}^{p}\right] \\
& -\int_{A^{-}} K(x)\left[\bar{F}\left(-a_{k}\right)-\bar{F}\left(\bar{u}_{k}\right)\right]-\int_{A^{+}} K(x)\left[\bar{F}\left(a_{k}\right)-\bar{F}\left(\bar{u}_{k}\right)\right] .
\end{aligned}
$$

The sign of the first three integrals in the last expression is non-positive. Moreover, for every $s \in \mathbb{R}$ complying with $|s| \in\left[a_{k}, b_{k}\right]$, we have $\bar{F}\left(\operatorname{sgn}(s) a_{k}\right)-\bar{F}(s)$ $=\bar{f}\left(\theta_{s}\right)\left(\operatorname{sgn}(s) a_{k}-s\right)$ for some $\theta_{s} \in \mathbb{R}$ with $\left|\theta_{s}\right| \in\left(a_{k}, b_{k}\right), \operatorname{sgn}\left(\theta_{s}\right)=\operatorname{sgn}(s)$. Taking into account $\left(f_{2}\right)$ and the fact that $\bar{f}$ is odd, we conclude that $\bar{F}\left(\operatorname{sgn}(s) a_{k}\right)$ $-\bar{F}(s) \geq 0$ for every $|s| \in\left[a_{k}, b_{k}\right]$, thus, the sign of the last two integrals are also non-positive. Consequently, $\bar{E}_{\tau}\left(w_{k}\right)-\bar{E}_{\tau}\left(\bar{u}_{k}\right) \leq 0$. On the other hand, $\bar{E}_{\tau}\left(\bar{u}_{k}\right)=\inf _{S_{k}} \bar{E}_{\tau}$ and $w_{k} \in S_{k}$. Therefore, every term in the right hand side of the above expression should vanish. In particular,

$$
\int_{A}\left|\nabla \bar{u}_{k}\right|^{p}=0 \text { and } \int_{A^{-}}\left[a_{k}^{p}-\left|\bar{u}_{k}\right|^{p}\right]=\int_{A^{+}}\left[a_{k}^{p}-\bar{u}_{k}^{p}\right]=0 .
$$

The first relation implies the existence of a positive measured subset $\tilde{A}$ of $A$ and a constant $\tilde{c} \in \mathbb{R}$ such that $\bar{u}_{k}=\tilde{c}$ on the set $\tilde{A}$. Then, either $\tilde{A} \subset A^{-}$or $\tilde{A} \subset A^{+}$. When $\tilde{A} \subset A^{-}$, then

$$
0=\int_{A^{-}}\left[a_{k}^{p}-\left|\bar{u}_{k}\right|^{p}\right] \leq \int_{\tilde{A}}\left[a_{k}^{p}-\left|\bar{u}_{k}\right|^{p}\right]=\left[a_{k}^{p}-|\tilde{c}|^{p}\right] \operatorname{meas}(\tilde{A})<0,
$$

contradiction. A similar argument can be applied when $\tilde{A} \subset A^{+}$, obtaining also a contradiction. Therefore, we necessarily have $A=\emptyset$.

Proposition 3.2 Let $\bar{u}_{k} \in S_{k}$ be such that $\bar{E}_{\tau}\left(\bar{u}_{k}\right)=\inf _{S_{k}} \bar{E}_{\tau}$. Then $\bar{u}_{k}$ is a local minimum point of $\bar{E}_{\tau}$ in $W_{G_{\tau}}^{1, p}\left(\mathbb{R}^{N}\right)$. 
Proof. Assume the contrary, i.e., there exists a sequence $\left\{u_{n}\right\} \subset W_{G_{\tau}}^{1, p}\left(\mathbb{R}^{N}\right)$ which converges to $\bar{u}_{k}$ and $\bar{E}_{\tau}\left(u_{n}\right)<\bar{E}_{\tau}\left(\bar{u}_{k}\right)=\inf _{S_{k}} \bar{E}_{\tau}$ for all $n \in \mathbb{N}$. In particular, this inequality implies that $u_{n}$ does not belong to the set $S_{k}$ for any $n \in \mathbb{N}$. On the other hand, since $u_{n} \rightarrow \bar{u}_{k}$ in $W_{G_{\tau}}^{1, p}\left(\mathbb{R}^{N}\right)$, then Morrey's theorem implies that $u_{n} \rightarrow \bar{u}_{k}$ in $L^{\infty}\left(\mathbb{R}^{N}\right)$ as well. In particular, for every $0<\varepsilon<b_{k}-a_{k}$, there exists $n_{\varepsilon} \in \mathbb{N}$ such that $\left\|u_{n}-\bar{u}_{k}\right\|_{\infty}<\varepsilon$ for every $n \geq n_{\varepsilon}$. By using Proposition 3.1 and taking into account the choice of the number $\varepsilon$, we conclude that $\left\|u_{n}\right\|_{\infty} \leq\left\|u_{n}-\bar{u}_{k}\right\|_{\infty}+\left\|\bar{u}_{k}\right\|_{\infty}<\varepsilon+a_{k}<b_{k}$, i.e., $u_{n} \in S_{k}$ for every $n \geq n_{\varepsilon}$, which contradicts the above statement.

Proposition 3.3 Let $\gamma_{k}=\inf _{S_{k}} \bar{E}_{\tau}=\bar{E}_{\tau}\left(\bar{u}_{k}\right)$. Then $\gamma_{k}<0$ for all $k \in \mathbb{N}$ and $\lim _{k \rightarrow \infty} \gamma_{k}=0$.

To prove Proposition 3.3, we construct a special class of functions with suitably prescribed symmetry properties, which is of interest in its own right as well.

Lemma 3.2 Let $0 \leq r<R$. For every $s>0, G \in \tilde{P}_{N}$ and $\tau \in N(G)$ there exist a function $u \in W_{G_{\tau}}^{1, p}\left(\mathbb{R}^{N}\right)$ and a $G_{\tau}$-invariant set $D \subset \mathbb{R}^{N}$ with meas $(D)>0$ such that

i) $\operatorname{supp} u \subset\left\{x \in \mathbb{R}^{N}: r / 2 \leq|x| \leq R\right\}$;

ii) $\|u\|_{\infty} \leq s$

iii) $|\nabla u(x)| \leq \frac{8 s}{R-r} \sqrt{N}$ for a.e. $x \in \mathbb{R}^{N}$;

iv) $|u(x)|=s$ for every $x \in D$.

Proof. Let $G=O\left(N_{1}\right) \times \cdots \times O\left(N_{k}\right) \in \tilde{P}_{N}$ with $N_{i}=N_{j}$ for some $i, j \in$ $\{1, \ldots, k\}, i \neq j$, and $\tau\left(x_{1}, \ldots, x_{i}, \ldots, x_{j}, \ldots, x_{k}\right)=\left(x_{1}, \ldots, x_{j}, \ldots, x_{i}, \ldots, x_{k}\right)$ for every $x=\left(x_{1}, \ldots, x_{i}, \ldots, x_{j}, \ldots, x_{k}\right) \in \mathbb{R}^{N_{1}} \times \ldots \times \mathbb{R}^{N_{i}} \times \ldots \times \mathbb{R}^{N_{j}} \times \ldots \times \mathbb{R}^{N_{k}}$. Let us denote by $x^{i j}$ the vector in $R^{N-2 N_{i}}$ which is obtained by omitting $x_{i}, x_{j} \in \mathbb{R}^{N_{i}}$ from the vector $x \in \mathbb{R}^{N}$. An explicit function that verifies the requirements is

$$
\begin{aligned}
u(x)= & u\left(x_{i}, x_{j}, x^{i j}\right) \\
= & \frac{32 s}{\sqrt{R^{2}-r^{2}}(R-r)} \operatorname{sgn}\left(\left|x_{i}\right|-\left|x_{j}\right|\right) \\
& \times\left(\operatorname { m i n } \left\{\frac{R-r}{4}-\max \left\{|| x_{i}|+| x_{j}\left|-\frac{R+3 r}{4}\right|,\right.\right.\right. \\
& \left.\left.\left.\quad||\left|x_{i}\right|-\left|x_{j}\right|\left|-\frac{R+3 r}{4}\right|\right\}, \frac{R-r}{8}\right\}\right)_{+} \\
& \times\left(\frac{\sqrt{R^{2}-r^{2}}}{2}-\max \left\{\left|x^{i j}\right|, \frac{\sqrt{R^{2}-r^{2}}}{4}\right\}\right)_{+},
\end{aligned}
$$


where $r_{+}=\max \{0, r\}$. Note that when $N=2 N_{i}$ (i.e. the vector $x^{i j}$ does not appear), we formally put $x^{i j}=0$ in the above expression.

One can easily check that $i), i i)$ and $i i i)$ are satisfied, $u \in W^{1, p}\left(\mathbb{R}^{N}\right)$, and $u$ is $G_{\tau}$-invariant. Now, let $D=D_{r, R}$ given as

$$
\begin{array}{r}
D=\left\{x=\left(x_{i}, x_{j}, x^{i j}\right) \in \mathbb{R}^{N}:|| x_{i}|+| x_{j}\left|-\frac{R+3 r}{4}\right| \leq \frac{R-r}{8},\right. \\
||\left|x_{i}\right|-\left|x_{j}\right|\left|-\frac{R+3 r}{4}\right| \leq \frac{R-r}{8}, \\
\left.\left|x^{i j}\right| \leq \frac{\sqrt{R^{2}-r^{2}}}{4}\right\} .
\end{array}
$$

The $G_{\tau}$-invariance of $D$ it follows from a geometrical reason. Moreover, meas $(D)$ $>0$ and for every $x \in D$ we have $u(x)=u\left(x_{i}, x_{j}, x^{i j}\right)=s \cdot \operatorname{sgn}\left(\left|x_{i}\right|-\left|x_{j}\right|\right)$, thus $i v)$.

Proof of Proposition 3.3. Let us fix $\theta \in(0,1)$. On account of hypothesis $\left(K_{1}\right)$, there exists a measurable set $M_{\theta} \subset \mathbb{R}^{N}$ with positive measure such that

$$
K(x)>\theta\|K\|_{\infty} \text { for a.e. } x \in M_{\theta} .
$$

Since the function $K$ is radial, we may assume that $M_{\theta}$ is an $O(N)$-invariant set, i.e., $g M_{\theta}=M_{\theta}$ for every $g \in O(N)$. In particular, we may fix an annulus (or a ball) with center in the origin which is included a.e. in $M_{\theta}$. More precisely, there are $0 \leq r_{\theta}<R_{\theta}$ so that meas $\left\{x \in \mathbb{R}^{N}: r_{\theta} / 2 \leq|x| \leq R_{\theta}, x \notin M_{\theta}\right\}=0$.

By $\left(f_{1}\right)$ there exist $l>0$ and $\varrho \in(0, \delta)$ such that $\bar{F}(s)=F(s)>-l|s|^{p}$ for every $s \in(-\varrho, \varrho)$. Let $D=D_{r_{\theta}, R_{\theta}} \subset \mathbb{R}^{N}$ be the set from (4) and fix a number $L>0$ large enough such that

$$
L \theta\|K\|_{\infty} \operatorname{meas}(D)-l\|K\|_{1}-c_{r_{\theta}, R_{\theta}} / p>0,
$$

where

$$
c_{r_{\theta}, R_{\theta}}:=\left(\left(\frac{8 \sqrt{N}}{R_{\theta}-r_{\theta}}\right)^{p}+1\right)\left(R_{\theta}^{N}-\left(r_{\theta} / 2\right)^{N}\right) \omega_{N} .
$$

Here, $\omega_{N}$ denotes the volume of the unit ball in $\mathbb{R}^{N}$. By using the right hand side of $\left(f_{1}\right)$, there exists a sequence $\left\{s_{k}\right\}_{k \in \mathbb{N}} \subset(0, \varrho)$ converging to zero, and $\bar{F}\left(s_{k}\right)=\bar{F}\left(-s_{k}\right)=F\left(s_{k}\right)=F\left(-s_{k}\right)>L s_{k}^{p}$.

Let $\left\{s_{n_{k}}\right\}_{k \in \mathbb{N}}$ be a decreasing subsequence of $\left\{s_{k}\right\}_{k \in \mathbb{N}}$ such that $s_{n_{k}} \leq b_{k}$ for every $k \in \mathbb{N}$. Now, we apply Lemma 3.2 for every $s_{n_{k}}>0, k \in \mathbb{N}$, obtaining the set $D$ (fixed already, which was possible since it depends only on $r=r_{\theta}$, $R=R_{\theta}, G \in \tilde{P}_{N}$ and $\tau \in N(G)$ but not from the elements $s_{n_{k}}$ ) and a sequence of functions $\left\{u_{k}\right\}_{k \in \mathbb{N}} \in W_{G_{\tau}}^{1, p}\left(\mathbb{R}^{N}\right)$ with the properties $\left.\left.i\right)-i v\right)$. By $\left.i i\right)$, we have $\left\|u_{k}\right\|_{\infty} \leq s_{n_{k}} \leq b_{k}$; therefore, $u_{k} \in S_{k}$. Moreover, using $\left.\left.i\right)-i i i\right)$ and the above 
notation, we have

$$
\left\|u_{k}\right\|_{W^{1, p}}^{p} \leq\left(\left(\frac{8 \sqrt{N}}{R_{\theta}-r_{\theta}}\right)^{p}+1\right)\left(R_{\theta}^{N}-\left(r_{\theta} / 2\right)^{N}\right) \omega_{N} s_{n_{k}}^{p}=c_{r_{\theta}, R_{\theta}} s_{n_{k}}^{p} .
$$

Note that $D \subset\left\{x \in \mathbb{R}^{N}: r_{\theta} / 2 \leq|x| \leq R_{\theta}\right\}$; thus, for a.e. $x \in D$ the inequality (5) can be applied. Using the fact that $\bar{F}$ is even and $i v$ ), one has

$$
\int_{D} K(x) \bar{F}\left(u_{k}(x)\right) d x=\int_{D} K(x) \bar{F}\left(s_{n_{k}}\right) d x \geq L \theta\|K\|_{\infty} \operatorname{meas}(D) s_{n_{k}}^{p} .
$$

On the other hand,

$$
\int_{\mathbb{R}^{N} \backslash D} K(x) \bar{F}\left(u_{k}(x)\right) d x \geq-\int_{\mathbb{R}^{N} \backslash D} K(x) l\left|u_{k}(x)\right|^{p} d x \geq-l\|K\|_{1} s_{n_{k}}^{p} .
$$

Combining the above relations with (6), we obtain

$$
\bar{E}_{\tau}\left(u_{k}\right) \leq\left(\frac{1}{p} c_{r_{\theta}, R_{\theta}}-L \theta\|K\|_{\infty} \operatorname{meas}(D)+l\|K\|_{1}\right) s_{n_{k}}^{p}<0 .
$$

It remains to prove that $\lim _{k \rightarrow \infty} \gamma_{k}=0$. The mean value theorem and Proposition 3.1 implies that

$$
\left|F\left(\bar{u}_{k}(x)\right)\right| \leq a_{k} \max _{\left[-a_{k}, a_{k}\right]}|f| \leq a_{k} \max _{\left[-a_{1}, a_{1}\right]}|f| \text { for every } x \in \mathbb{R}^{N} .
$$

Then

$$
0>\gamma_{k}=\frac{1}{p}\left\|\bar{u}_{k}\right\|_{W^{1, p}}^{p}-\int_{\mathbb{R}^{N}} K(x) F\left(\bar{u}_{k}(x)\right) d x \geq-a_{k}\|K\|_{1} \max _{\left[a_{1}, a_{1}\right]}|f| .
$$

Since the sequence $\left\{a_{k}\right\}_{k \in \mathbb{N}}$ tends to zero, the same is true for $\left\{\gamma_{k}\right\}_{k \in \mathbb{N}}$.

Now, we are in the position to prove the first part of our main result, i.e., to guarantee infinitely many non-radial, sign-changing solutions of $(\mathrm{P})$ which belong to the space $W_{G_{\tau}}^{1, p}\left(\mathbb{R}^{N}\right)$.

Proof of Theorem 2.1 (first part). Due to Proposition 3.3, there are infinitely many pairwise distinct nonzero local minima of $\bar{E}_{\tau}$ (up to a subsequence, we will keep the same notation $\bar{u}_{k}$ as before). Since $\bar{E}$ is even and $K$ radial, $\bar{E}$ is $G_{\tau}$-invariant, i.e., $\bar{E}(h u)=\bar{E}(u)$ for every $h \in G_{\tau}, u \in W^{1, p}\left(\mathbb{R}^{N}\right)$. Thus, one can apply the principle of symmetric criticality of Palais [18], obtaining that the local minima of $\bar{E}_{\tau}=\bar{E}_{\mid W_{G_{\tau}}^{1, p}\left(\mathbb{R}^{N}\right)}$ are actually critical points of $\bar{E}$, thus, weak solutions of the problem

$$
\left\{\begin{array}{l}
-\triangle_{p} u+|u|^{p-2} u=K(x) \bar{f}(u), \quad x \in \mathbb{R}^{N}, \\
u \in W^{1, p}\left(\mathbb{R}^{N}\right) .
\end{array}\right.
$$


Since $\left\|\bar{u}_{k}\right\|_{\infty} \leq a_{k} \leq \delta$ for every $k \in \mathbb{N}$, then $\bar{f}\left(\bar{u}_{k}(x)\right)=f\left(\bar{u}_{k}(x)\right)$ for every $x \in \mathbb{R}^{N}$. Consequently, the elements $\bar{u}_{k}, k \in \mathbb{N}$, are weak solutions not only for $(\overline{\mathrm{P}})$ but also for $(\mathrm{P})$. Due to Remark 3.1 , the elements $\bar{u}_{k}, k \in \mathbb{N}$, are non-radial and change their signs. Finally, the convergence of the sequence $\left\{a_{k}\right\}_{k \in \mathbb{N}}$ to zero implies that $\lim _{k \rightarrow \infty}\left\|\bar{u}_{k}\right\|_{\infty}=0$, which concludes the proof of the first part of Theorem 2.1 .

\section{Construction of special subspaces of $W^{1, p}\left(\mathbb{R}^{N}\right)$; sequences of solutions for $(\mathrm{P})$ with mutually different symmetries}

In this section we will give a possible answer to the question formulated in the Introduction, i.e., to describe as many sequences of non-radial, sign-changing weak solutions of $(\mathrm{P})$ as we can having the properties that elements in different sequences are mutually distinguished by their symmetry properties. Now, we are going to construct $s_{N}$ special subspaces of $W^{1, p}\left(\mathbb{R}^{N}\right)$ such that:

- For every subspace one can apply the arguments from Section 3, obtaining infinitely many non-radial, sign-changing weak solutions of $(\mathrm{P})$;

- The pairwise intersection of the subspaces contains only the 0 element. (As a consequence, the sequences belonging to different subspaces cannot be compared by symmetrical point of view.)

Let $G=O\left(N_{1}^{G}\right) \times \ldots \times O\left(N_{n}^{G}\right) \in P_{N}$ and $H=O\left(N_{1}^{H}\right) \times \ldots \times O\left(N_{m}^{H}\right) \in P_{N}$, and define the set of coincidences of the partition of $N$ with respect to the groups $G$ and $H$ by

$C(G, H)=\left\{(i, j) \in\{1, \ldots, n\} \times\{1, \ldots, m\}: N_{1}^{G}+\ldots+N_{i}^{G}+N_{j}^{H}+\ldots+N_{m}^{H}=N\right\}$.

Moreover, let us denote by $\langle G, H\rangle$ the subgroup of $O(N)$ generated (topologically) by $G$ and $H$, and $S_{\rho}\left(\mathbb{R}^{m}\right) \subset \mathbb{R}^{m}$ be the $(m-1)$-dimensional sphere with radius $\rho>0,(m=2, \ldots, N)$. When $\rho=1$, we simply denote by $S\left(\mathbb{R}^{m}\right) \subset \mathbb{R}^{m}$ the $(m-1)$-dimensional unit sphere.

Lemma 4.1 Let $G, H \in P_{N}$. If $C(G, H)=\emptyset$ then $\langle G, H\rangle$ acts transitively on $S\left(\mathbb{R}^{N}\right)$, i.e., the $\langle G, H\rangle$-orbit of every unit vector in $\mathbb{R}^{N}$ is $S\left(\mathbb{R}^{N}\right)$.

Proof. Let $G=O\left(N_{1}^{G}\right) \times \ldots \times O\left(N_{n}^{G}\right) \in P_{N}$ and $H=O\left(N_{1}^{H}\right) \times \ldots \times O\left(N_{m}^{H}\right) \in$ $P_{N}$. Without loosing the generality, we may assume that $N_{1}^{G}<N_{1}^{H}$ (these two numbers cannot be equal since $C(G, H)=\emptyset)$. Using again $C(G, H)=\emptyset$, there is a (unique) number $i_{1} \in\{1, \ldots, n-1\}$ such that

$$
N_{1}^{G}+\ldots+N_{i_{1}}^{G}<N_{1}^{H}<N_{1}^{G}+\ldots+N_{i_{1}+1}^{G}
$$


Let us denote by

$$
\tilde{V}_{1}=\mathbb{R}^{N_{1}^{G}+\ldots+N_{i_{1}}^{G}}, \tilde{V}_{2}=\mathbb{R}^{N_{i_{1}+1}^{G}}, V_{1}=\mathbb{R}^{N_{1}^{H}}, V_{2}=\mathbb{R}^{N_{1}^{G}+\ldots+N_{i_{1}+1}^{G}-N_{1}^{H}},
$$

and let $V=\tilde{V}_{1} \times \tilde{V}_{2}=V_{1} \times V_{2}=\mathbb{R}^{N_{1}^{G}+\ldots+N_{i_{1}+1}^{G}}$.

We first claim that $\langle G, H\rangle$ acts transitively on $S(V)$, i.e., for every $v, w \in$ $S(V)$ there exists $\sigma_{v, w} \in\langle G, H\rangle$ such that

$$
\sigma_{v, w}(v, 0)=(w, 0)
$$

where the element 0 belongs to $\mathbb{R}^{N-\operatorname{dim} V}$. (If $N=\operatorname{dim} V$ the element 0 vanishes in (7) and we are done.) In order to prove (7) it is enough to show that for every $v_{1} \in S\left(V_{1}\right)=S\left(\mathbb{R}^{N_{1}^{H}}\right)$ and $w \in S(V)$ there are $g_{w} \in G$ and $h_{w} \in H$ such that

$$
g_{w} h_{w}\left(\left(v_{1}, 0\right), 0\right)=(w, 0),
$$

where $\left(v_{1}, 0\right) \in S(V)$. Indeed, having a similar relation $g_{v} h_{v}\left(\left(v_{1}, 0\right), 0\right)=(v, 0)$ for some $g_{v} \in G$ and $h_{v} \in H$, we deduce that $\sigma_{v, w}=g_{w} h_{w}\left(g_{v} h_{v}\right)^{-1} \in\langle G, H\rangle$ fulfills (7).

Therefore, we prove (8), fixing arbitrarily $v_{1} \in S\left(V_{1}\right)$ and $w=$ $\left(w_{11}, w_{12}, w_{2}\right) \in S\left(\tilde{V}_{1} \times \mathbb{R}^{N_{1}^{H}-\operatorname{dim} \tilde{V}_{1}} \times V_{2}\right)=S(V)$. Since $O\left(N_{1}^{H}\right)$ acts transitively on $S\left(V_{1}\right)=S\left(\mathbb{R}^{N_{1}^{H}}\right)$, for every $\tilde{w}_{12} \in \mathbb{R}^{N_{1}^{H}-\operatorname{dim} \tilde{V}_{1}}$ with the property $\left(w_{11}, \tilde{w}_{12}\right) \in S\left(V_{1}\right)$, there exists an $h_{1} \in O\left(N_{1}^{H}\right)$ such that

$$
h_{1} v_{1}=\left(w_{11}, \tilde{w}_{12}\right) \text {. }
$$

Note that $1=\left|w_{11}\right|^{2}+\left|\tilde{w}_{12}\right|^{2}$ and $1=\left|w_{11}\right|^{2}+\left|w_{12}\right|^{2}+\left|w_{2}\right|^{2}$. Therefore,

$$
\left|\tilde{w}_{12}\right|^{2}=\left|w_{12}\right|^{2}+\left|w_{2}\right|^{2} \text {. }
$$

If $\tilde{w}_{12}=0$, on account of $(10)$, we have $w=\left(w_{11}, 0,0\right)$. In this case, $(8)$ is clearly verified, choosing $g_{w}=\operatorname{Id}_{\mathbb{R}^{N}} \in G$ and $h_{w}=h_{1} \times \operatorname{Id}_{\mathbb{R}^{N-N_{1}^{H}}} \in H$.

On the other hand, if $\tilde{w}_{12} \neq 0$, let $\rho=\left|\tilde{w}_{12}\right|>0$. Since $O\left(\mathbb{R}^{N_{i_{1}+1}^{G}}\right)$ acts transitively on $S\left(\tilde{V}_{2}\right)$, it acts transitively on $S_{\rho}\left(\tilde{V}_{2}\right)$ as well. Consequently by $(10)$, there exists $g_{1} \in O\left(N_{i_{1}+1}^{G}\right)$ such that

$$
g_{1}\left(\tilde{w}_{12}, 0\right)=\left(w_{12}, w_{2}\right) \in S_{\rho}\left(\tilde{V}_{2}\right)=S_{\rho}\left(\mathbb{R}^{N_{i_{1}+1}^{G}}\right) .
$$

Taking

and

$$
g_{w}=\operatorname{Id}_{\tilde{V}_{1}} \times g_{1} \times \operatorname{Id}_{\mathbb{R}^{N-\operatorname{dim} V}} \in G
$$

$$
h_{w}=h_{1} \times \operatorname{Id}_{\mathbb{R}^{N-N_{1}^{H}}} \in H,
$$

on account of (9) and (11), one has

$$
g_{w} h_{w}\left(\left(v_{1}, 0\right), 0\right)=g_{w}\left(w_{11}, \tilde{w}_{12}, 0\right)=\left(w_{11}, w_{12}, w_{2}, 0\right)=(w, 0),
$$

which proves relation (8), thus (7). 
If $N_{1}^{G}+\ldots+N_{i_{1}+1}^{G}=N$ then the statement of our lemma is concluded. Otherwise, if $N_{1}^{G}+\ldots+N_{i_{1}+1}^{G}<N$, using $C(G, H)=\emptyset$, we may fix a number $j_{1} \in\{1, \ldots, m-1\}$ such that

$$
N_{1}^{H}+\ldots+N_{j_{1}}^{H}<N_{1}^{G}+\ldots+N_{i_{1}+1}^{G}<N_{1}^{H}+\ldots+N_{j_{1}+1}^{H} .
$$

Now, we may continue inductively the above arguments arriving to $N$ as the right hand side of the above inequality for $H$ (or its analog for $G$ ). Consequently, for every elements $v, w \in S\left(\mathbb{R}^{N}\right)$ one can find finitely many elements $g_{1}, g_{2}, \ldots \in G$ and $h_{1}, h_{2}, \ldots \in H$ such that $\left(g_{1} h_{1} g_{2} h_{2} \ldots\right) v=w$, which concludes our proof.

Corollary 4.1 Let $G, H \in P_{N}$ and let $u: \mathbb{R}^{N} \rightarrow \mathbb{R}$ be a G-invariant and $H$-invariant function. If $C(G, H)=\emptyset$ then $u$ is radial.

Proof. It is clear that $u$ is also $\langle G, H\rangle$-invariant. On the other hand, Lemma 4.1 implies that the $\langle G, H\rangle$-orbit of every element $v \in \mathbb{R}^{N} \backslash\{0\}$ is $S_{|v|}\left(\mathbb{R}^{N}\right)$. In particular, the function $u$ is constant on the $\langle G, H\rangle$-orbit of every element $v \in \mathbb{R}^{N} \backslash\{0\}$, i.e., it depends only in $|v|$ which means that $u$ is radial.

Remark 4.1 Ultimately, it seems that $C(G, H)=\emptyset$ if and only if $\langle G, H\rangle=$ $O(N)$. The "if" part is obvious. Indeed, let us assume that $\langle G, H\rangle=O(N)$ and by contradiction, $C(G, H) \neq \emptyset$. Then, for some $(i, j) \in\{1, \ldots, n\} \times\{1, \ldots, m\}$ we have $O(N)=\langle G, H\rangle \subseteq O\left(N_{1}^{G}+\ldots+N_{i}^{G}\right) \times O\left(N_{j}^{H}+\ldots+N_{m}^{H}\right) O(N)$, contradiction. The "only if" part should follow from Lemma 4.1 and the classification of all linear orthogonal groups which act transitively on the sphere, see Borel [7].

Proposition 4.1 Let $G, H \in \tilde{P}_{N}$ and $\tau \in N(G), \gamma \in N(H)$ arbitrarily fixed. If $C(G, H)=\emptyset$ then

$$
W_{G_{\tau}}^{1, p}\left(\mathbb{R}^{N}\right) \cap W_{H_{\gamma}}^{1, p}\left(\mathbb{R}^{N}\right)=\{0\}
$$

Proof. Let $u \in W_{G_{\tau}}^{1, p}\left(\mathbb{R}^{N}\right) \cap W_{H_{\gamma}}^{1, p}\left(\mathbb{R}^{N}\right)$. In particular, $u$ is both $G$-invariant and $H$-invariant. On account of Corollary 4.1, the function $u$ is radial. On the other hand, since $u$ is $G_{\tau}$-invariant, we have $u(x)=-u\left(\tau^{-1} x\right)$ for every $x \in \mathbb{R}^{N}$. Since $|x|=\left|\tau^{-1} x\right|$, the function $u$ is identically 0 .

Proposition 4.2 Let $N \geq 4, N \neq 5$, and

$$
G_{l}^{N}=O(l+1) \times O(N-2 l-2) \times O(l+1) \in \tilde{P}_{N}, \quad l \in\left\{1, \ldots, s_{N}\right\} .
$$

Then $C\left(G_{k}^{N}, G_{l}^{N}\right)=\emptyset$ for every $k, l \in\left\{1, \ldots, s_{N}\right\}, k \neq l$.

Proof. Notice that $N-2 l-2$ may become 0 ; in such case, we refer to $G_{l}^{N}=$ $O(l+1) \times O(l+1)$. Now, let us fix $k, l \in\left\{1, \ldots, s_{N}\right\}, k \neq l$. Note that at most 
9 possibilities there exist to obtain elements of $C\left(G_{k}^{N}, G_{l}^{N}\right)$; indeed, since the partitions of $N$ have the forms

$$
\begin{aligned}
N & =(k+1)+(N-2 k-2)+(k+1) \\
& =(l+1)+(N-2 l-2)+(l+1),
\end{aligned}
$$

the possible elements of the set $C\left(G_{k}^{N}, G_{l}^{N}\right)$ are $(i, j) \in\{1,2,3\} \times\{1,2,3\}$.

First, let us assume that $N-2 k-2 \neq 0$ and $N-2 l-2 \neq 0$. One can easily observe that neither the elements of the form $(i, 1), i \in\{1,2,3\}$, nor $(3, j)$, $j \in\{1,2,3\}$, cannot belong to the set $C\left(G_{k}^{N}, G_{l}^{N}\right)$. Thus, we have the following remaining four cases:

I.) Suppose that $(i, j)=(1,2) \in C\left(G_{k}^{N}, G_{l}^{N}\right)$. Then $(k+1)+(N-2 l-2)+$ $(l+1)=N$ which contradicts the fact that $k \neq l$.

II.) Suppose that $(i, j)=(2,2) \in C\left(G_{k}^{N}, G_{l}^{N}\right)$. Then $(k+1)+(N-2 k-2)+$ $(N-2 l-2)+(l+1)=N$, i.e., $N=k+l+2$. Since $k \neq l$, we have $k+l \leq 2 s_{N}-1$. Consequently, $N=k+l+2 \leq 2 s_{N}+1<N$, contradiction.

III.) $(i, j)=(2,3)$ works similarly as I.).

IV.) $(i, j)=(1,3)$ reduces to a similar discussion as II.).

Therefore, $C\left(G_{k}^{N}, G_{l}^{N}\right)=\emptyset$.

Now, assume without losing the generality that $N-2 l-2=0$. Then $k \neq$ $l=N / 2-1$ and the partitions of $N$ have the forms

$$
\begin{aligned}
N & =(k+1)+(N-2 k-2)+(k+1) \\
& =N / 2+N / 2 .
\end{aligned}
$$

In particular, possible elements of $C\left(G_{k}^{N}, G_{l}^{N}\right)$ are $(i, j) \in\{1,2,3\} \times\{1,2\}$. Similarly as before, neither the elements of the form $(i, 1), i \in\{1,2,3\}$, nor $(3, j)$, $j \in\{1,2\}$, cannot belong to the set $C\left(G_{k}^{N}, G_{l}^{N}\right)$. If $(1,2) \in C\left(G_{k}^{N}, G_{l}^{N}\right)$, then $(k+1)+N / 2=N$, i.e., $k=l$, contradiction. Finally, if $(2,2) \in C\left(G_{k}^{N}, G_{l}^{N}\right)$, then $(k+1)+(N-2 k-2)+N / 2=N$, i.e., $k=l$, contradiction. Consequently, $C\left(G_{k}^{N}, G_{l}^{N}\right)=\emptyset$.

Proof of Theorem 2.1 (concluded). On account of Propositions 4.2 and 4.1, respectively, for every $N \geq 4, N \neq 5$, one can construct $s_{N} \in \mathbb{N}$ groups $G_{l}^{N} \in \tilde{P}_{N}$, $l \in\left\{1, \ldots, s_{N}\right\}$ such that for every $\tau_{l}^{N} \in N\left(G_{l}^{N}\right)$ the pairwise intersection of different spaces of the form $W_{\left(G_{l}^{N}\right)_{\tau_{l}^{N}}^{1, p}}\left(\mathbb{R}^{N}\right)$ contains only the 0 element. Applying

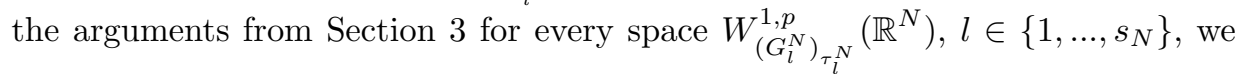
are able to guarantee $s_{N}$ sequences of non-radial, sign-changing weak solutions of (P) with the property that different sequences are mutually distinguished by their symmetry properties. This concludes the proof of Theorem 2.1.

Remark 4.2 In order to be more explicit, in Table 1 (see below) we give the description of the groups $G_{l}^{N}$ and possible choices of $\tau_{l}^{N} \in N\left(G_{l}^{N}\right), l \in\left\{1, \ldots, s_{N}\right\}$ for the space dimensions $N=4, \ldots, 13$. 
Even if we exploit all the possibilities of the choice of $\tau_{l}^{N} \in N\left(G_{l}^{N}\right)$, $l \in\left\{1, \ldots, s_{N}\right\}$, in general, the number of sequences of solutions distinguished by their symmetry properties cannot be increased by the aforementioned way. To see this, let us consider the case $N=6$. Note that $N\left(G_{2}^{6}\right)=\left\{\tau_{2}^{6}\right\}$ (see Table 1 ), and $N\left(G_{1}^{6}\right)=\left\{\gamma_{1}, \gamma_{2}, \gamma_{3}\right\}$, where $\gamma_{1}\left(x_{1}, x_{2}, x_{3}\right)=\left(x_{1}, x_{3}, x_{3}\right), \gamma_{2}\left(x_{1}, x_{2}, x_{3}\right)=$ $\left(x_{3}, x_{2}, x_{1}\right), \gamma_{3}\left(x_{1}, x_{2}, x_{3}\right)=\left(x_{2}, x_{1}, x_{3}\right)$ with $x_{i} \in \mathbb{R}^{2}, i \in\{1,2,3\}$. Due to Proposition 4.1,

$$
W_{\left(G_{1}^{6}\right)_{\gamma_{i}}}^{1, p}\left(\mathbb{R}^{6}\right) \cap W_{\left(G_{2}^{6}\right)_{\tau_{2}^{6}}^{1, p}}\left(\mathbb{R}^{6}\right)=\{0\}
$$

for every $i \in\{1,2,3\}$. However,

$$
\bigcap_{i=1}^{3} W_{\left(G_{1}^{6}\right)_{\gamma_{i}}}^{1, p}\left(\mathbb{R}^{6}\right) \neq\{0\} .
$$

Indeed, the function $u: \mathbb{R}^{6} \rightarrow \mathbb{R}$ defined by

$$
u\left(x_{1}, x_{2}, x_{3}\right)=\left(\left|x_{1}\right|-\left|x_{2}\right|\right)\left(\left|x_{2}\right|-\left|x_{3}\right|\right)\left(\left|x_{3}\right|-\left|x_{1}\right|\right)\left(1-\left|x_{1}\right|-\left|x_{2}\right|-\left|x_{3}\right|\right)_{+},
$$

\begin{tabular}{|c|c|c|c|}
\hline $\bar{N}$ & $N$ & $l=P_{N} ; l \in\{1$, & le choice of $\tau_{l}^{N} \in N(C$ \\
\hline 4 & & $G_{1}^{4}=O(2) \times O(2)$ & $\tau_{1}^{4}\left(x_{1}, x_{2}\right)=\left(x_{2}, x_{1}\right) ; x_{1}, x_{2} \in \mathbb{R}^{2}$ \\
\hline 5 & 0 & - & - \\
\hline 6 & 2 & $\begin{array}{c}G_{1}^{6}=O(2) \times O(2) \times O(2) \\
G_{2}^{6}=O(3) \times O(3)\end{array}$ & $\begin{array}{c}\tau_{1}^{6}\left(x_{1}, x_{2}, x_{3}\right)=\left(x_{3}, x_{2}, x_{1}\right) ; x_{1}, x_{2}, x_{3} \in \mathbb{R}^{2} \\
\tau_{2}^{6}\left(x_{1}, x_{2}\right)=\left(x_{2}, x_{1}\right) ; x_{1}, x_{2} \in \mathbb{R}^{3}\end{array}$ \\
\hline 7 & 1 & $G_{1}^{7}=O(2) \times O(3) \times O(2)$ & $\left(x_{1}, x_{2}, x_{3}\right)=\left(x_{3}, x_{2}, x_{1}\right) ; x_{1}, x_{3} \in \mathbb{R}^{2}, x_{2} \in \mathbb{R}^{3}$ \\
\hline 8 & 3 & $\begin{array}{c}G_{1}^{8}=O(2) \times O(4) \times O(2) \\
G_{2}^{8}=O(3) \times O(2) \times O(3) \\
G_{3}^{8}=O(4) \times O(4)\end{array}$ & $\begin{array}{c}\tau_{1}^{8}\left(x_{1}, x_{2}, x_{3}\right)=\left(x_{3}, x_{2}, x_{1}\right) ; x_{1}, x_{3} \in \mathbb{R}^{2}, x_{2} \in \mathbb{R}^{4} \\
\tau_{2}^{8}\left(x_{1}, x_{2}, x_{3}\right)=\left(x_{3}, x_{2}, x_{1}\right) ; x_{1}, x_{3} \in \mathbb{R}^{3}, x_{2} \in \mathbb{R}^{2} \\
\tau_{3}^{8}\left(x_{1}, x_{2}\right)=\left(x_{2}, x_{1}\right) ; x_{1}, x_{2} \in \mathbb{R}^{4}\end{array}$ \\
\hline 9 & 2 & $\begin{aligned} G_{1}^{9} & =O(2) \times O(5) \times O(2) \\
G_{2}^{9} & =O(3) \times O(3) \times O(3)\end{aligned}$ & $\begin{array}{c}\tau_{1}^{9}\left(x_{1}, x_{2}, x_{3}\right)=\left(x_{3}, x_{2}, x_{1}\right) ; x_{1}, x_{3} \in \mathbb{R}^{2}, x_{2} \in \mathbb{R}^{5} \\
\tau_{2}^{9}\left(x_{1}, x_{2}, x_{3}\right)=\left(x_{2}, x_{1}, x_{3}\right) ; x_{1}, x_{2}, x_{3} \in \mathbb{R}^{3}\end{array}$ \\
\hline 10 & 4 & $\begin{array}{c}G_{1}^{10}=O(2) \times O(6) \times O(2) \\
G_{2}^{10}=O(3) \times O(4) \times O(3) \\
G_{3}^{10}=O(4) \times O(2) \times O(4) \\
G_{4}^{10}=O(5) \times O(5)\end{array}$ & $\begin{array}{c}\tau_{1}^{10}\left(x_{1}, x_{2}, x_{3}\right)=\left(x_{3}, x_{2}, x_{1}\right) ; x_{1}, x_{3} \in \mathbb{R}^{2}, x_{2} \in \mathbb{R}^{6} \\
\tau_{2}^{10}\left(x_{1}, x_{2}, x_{3}\right)=\left(x_{3}, x_{2}, x_{1}\right) ; x_{1}, x_{3} \in \mathbb{R}^{3}, x_{2} \in \mathbb{R}^{4} \\
\tau_{3}^{10}\left(x_{1}, x_{2}, x_{3}\right)=\left(x_{3}, x_{2}, x_{1}\right) ; x_{1}, x_{3} \in \mathbb{R}^{4}, x_{2} \in \mathbb{R}^{2} \\
\tau_{4}^{10}\left(x_{1}, x_{2}\right)=\left(x_{2}, x_{1}\right) ; x_{1}, x_{2} \in \mathbb{R}^{5}\end{array}$ \\
\hline 11 & 3 & $\begin{array}{l}G_{1}^{11}=O(2) \times O(7) \times O(2) \\
G_{2}^{11}=O(3) \times O(5) \times O(3) \\
G_{3}^{11}=O(4) \times O(3) \times O(4)\end{array}$ & $\begin{array}{l}\tau_{1}^{11}\left(x_{1}, x_{2}, x_{3}\right)=\left(x_{3}, x_{2}, x_{1}\right) ; x_{1}, x_{3} \in \mathbb{R}^{2}, x_{2} \in \mathbb{R}^{7} \\
\tau_{2}^{11}\left(x_{1}, x_{2}, x_{3}\right)=\left(x_{3}, x_{2}, x_{1}\right) ; x_{1}, x_{3} \in \mathbb{R}^{3}, x_{2} \in \mathbb{R}^{5} \\
\tau_{3}^{11}\left(x_{1}, x_{2}, x_{3}\right)=\left(x_{3}, x_{2}, x_{1}\right) ; x_{1}, x_{3} \in \mathbb{R}^{4}, x_{2} \in \mathbb{R}^{3}\end{array}$ \\
\hline 12 & 5 & $\begin{array}{c}G_{1}^{12}=O(2) \times O(8) \times O(2) \\
G_{2}^{12}=O(3) \times O(6) \times O(3) \\
G_{3}^{12}=O(4) \times O(4) \times O(4) \\
G_{4}^{12}=O(5) \times O(2) \times O(5) \\
G_{5}^{12}=O(6) \times O(6)\end{array}$ & $\begin{array}{c}\tau_{1}^{12}\left(x_{1}, x_{2}, x_{3}\right)=\left(x_{3}, x_{2}, x_{1}\right) ; x_{1}, x_{3} \in \mathbb{R}^{2}, x_{2} \in \mathbb{R}^{8} \\
\tau_{2}^{12}\left(x_{1}, x_{2}, x_{3}\right)=\left(x_{3}, x_{2}, x_{1}\right) ; x_{1}, x_{3} \in \mathbb{R}^{3}, x_{2} \in \mathbb{R}^{6} \\
\tau_{3}^{12}\left(x_{1}, x_{2}, x_{3}\right)=\left(x_{2}, x_{1}, x_{3}\right) ; x_{1}, x_{2}, x_{3} \in \mathbb{R}^{4} \\
\tau_{4}^{12}\left(x_{1}, x_{2}, x_{3}\right)=\left(x_{3}, x_{2}, x_{1}\right) ; x_{1}, x_{3} \in \mathbb{R}^{5}, x_{2} \in \mathbb{R}^{2} \\
\tau_{5}^{12}\left(x_{1}, x_{2}\right)=\left(x_{2}, x_{1}\right) ; x_{1}, x_{2} \in \mathbb{R}^{6}\end{array}$ \\
\hline 13 & 4 & $\begin{array}{l}G_{1}^{13}=O(2) \times O(9) \times O(2) \\
G_{2}^{13}=O(3) \times O(7) \times O(3) \\
G_{3}^{13}=O(4) \times O(5) \times O(4) \\
G_{4}^{13}=O(5) \times O(3) \times O(5)\end{array}$ & $\begin{array}{l}\tau_{1}^{13}\left(x_{1}, x_{2}, x_{3}\right)=\left(x_{3}, x_{2}, x_{1}\right) ; x_{1}, x_{3} \in \mathbb{R}^{2}, x_{2} \in \mathbb{R}^{9} \\
\tau_{2}^{13}\left(x_{1}, x_{2}, x_{3}\right)=\left(x_{3}, x_{2}, x_{1}\right) ; x_{1}, x_{3} \in \mathbb{R}^{3}, x_{2} \in \mathbb{R}^{7} \\
\tau_{3}^{13}\left(x_{1}, x_{2}, x_{3}\right)=\left(x_{3}, x_{2}, x_{1}\right) ; x_{1}, x_{3} \in \mathbb{R}^{4}, x_{2} \in \mathbb{R}^{5} \\
\tau_{4}^{13}\left(x_{1}, x_{2}, x_{3}\right)=\left(x_{3}, x_{2}, x_{1}\right) ; x_{1}, x_{3} \in \mathbb{R}^{5}, x_{2} \in \mathbb{R}^{3}\end{array}$ \\
\hline
\end{tabular}

$x_{i} \in \mathbb{R}^{2}, i \in\{1,2,3\}$, does belong to the set $\bigcap_{i=1}^{3} W_{\left(G_{1}^{6}\right)}^{1, p}\left(\mathbb{R}^{6}\right)$. Therefore, one could happen that certain elements belong to several sequences of solutions.

Table 1 
Remark 4.3 Fix $l \in\left\{1, \ldots, s_{N}\right\}$ and $\tau_{l}^{N} \in N\left(G_{l}^{N}\right)$, defined by $\tau_{l}^{N}\left(x_{1}, x_{2}, x_{3}\right)=$ $\left(x_{3}, x_{2}, x_{1}\right)$ for every $\left(x_{1}, x_{2}, x_{3}\right) \in \mathbb{R}^{l+1} \times \mathbb{R}^{N-2 l-2} \times \mathbb{R}^{l+1}$. Let us consider the 'diagonal' set

$$
D_{l}^{N}=\left\{x=\left(x_{1}, x_{2}, x_{3}\right) \in \mathbb{R}^{l+1} \times \mathbb{R}^{N-2 l-2} \times \mathbb{R}^{l+1}:\left|x_{1}\right|=\left|x_{3}\right|\right\} .
$$

If $\left\{u_{k}^{l}\right\}_{k \in \mathbb{N}} \subset W_{\left(G_{l}^{N}\right)_{\tau_{l}^{N}}^{1, p}}\left(\mathbb{R}^{N}\right)$ is a sequence of solutions from Theorem 2.1, then $u_{k}^{l}(x)=0$ for every $x \in D_{l}^{N}, k \in \mathbb{N}$. This fact follows from (2).

\section{References}

[1] T. BARTSCH, Critical point theory on partially ordered Hilbert spaces, J. Funct. Anal. 186 (2001), 117-152.

[2] T. BARTSCH, Z. LIU and T. WETH, Nodal solutions of a $p$-Laplacian eqaution, Proc. London Math. Soc. 91 (2005), 129-152.

[3] T. BARTSCH, Z. LIU and T. WETH, Sign changing solutions of superlinear Schrödinger equations, Comm. Partial Differential Equations 29 (2004), $25-42$.

[4] T. BARTSCH, M. SCHNEIDER and T. WETH, Multiple solutions of a critical polyharmonic equation, J. Reine Angew. Math. 571 (2004), 131-143.

[5] T. BARTSCH and M. WILLEM, Infinitely many non-radial solutions of an Euclidean scalar field equations, J. Funct. Anal. 117 (1993), 447-460 .

[6] V. BENCI, D. FORTUNAT and L. PISANI, Solitons in several space dimensions: Derrick's problem and infinitely many solutions, Arch. Ration. Mech. Anal. 154 (2000), 297-324.

[7] A. BOREL, Le plan projectif des octaves et les sphères comme espaces homogènes, C. R. Acad. Sci. Paris 230 (1950), 1378-1380.

[8] H. BRÉZIS, Analyse Fonctionnelle - Théorie et Applications, Masson, Paris, 1992.

[9] P. DRÁBEK and Y. X. HUANG, Multiple positive solutions of quasilinear elliptic equations in $\mathbb{R}^{n}$, Nonlinear Anal. 37 (1999), 457-466.

[10] P. DRÁBEK and C. G. SIMADER, Nonlinear eigenvalue problems for quasilinear equations in unbounded domains, Math. Nachr. 203 (1999), 5-30.

[11] A. KRISTÁLY, Infinitely many solutions for a differential inclusion problem in $\mathbb{R}^{N}$, J. Differential Equations 220 (2006), 511-530. 
[12] A. KRISTÁLY, GH. MOROCSANU and S. TERSIAN, Quasilinear elliptic problems in $\mathbb{R}^{N}$ involving oscillatory nonlinearities, J. Differential Equations 235 (2007), 366-375.

[13] A. KRISTÁLY and CS. VARGA, On a class of quasilinear eigenvalue problems in $\mathbb{R}^{N}$, Math. Nachr. 278 (2005), 1756-1765.

[14] G. LI and S. YAN, Eigenvalue problems for quasilinear elliptic equations on $\mathbb{R}^{N}$, Comm. Partial Differential Equations 14 (1989), 1291-1314.

[15] S. J. LI and Z.-Q. WANG, Ljusternik-Schnirelman theory in partially ordered Hilbert spaces, Trans. Amer. Math. Soc. 354 (2002), 3207-3227.

[16] P.-L. LIONS, Symétrie et compacité dans les espaces de Sobolev, J. Funct. Anal. 49 (1982), 315-334.

[17] M. MARCUS and V. MIZEL, Every superposition operator mapping one Sobolev space into another is continuous, J. Funct. Anal. 33 (1979), 217-229.

[18] R. PALAIS, The principle of symmetric criticality, Comm. Math. Phys. 69 (1979), 19-30.

[19] B. RICCERI, Infinitely many solutions of the Neumann problem for elliptic equations involving the $p$-Laplacian, Bull. London Math. Soc. 33 (2001), $331-340$.

[20] J. SAINT RAYMOND, On the multiplicity of the solutions of the equation $-\Delta u=\lambda f(u), J$. Differential Equations 180 (2002), 65-88.

[21] Z.-Q. WANG, Nonlinear boundary value problems with concave nonlinearities near the origin, NoDEA Nonlinear Differential Equations Appl. 8 (1) (2001) $15-33$.

[22] Z. ZHANG, J. CHEN and S. LI, Construction of pseudo-gradient vector field and sign-changing multiple solutions involving $p$-Laplacian, J. Differential Equations 201 (2004), 287-303.

Received 6 September 2006; accepted 28 May 2007;

published Online First 10 January 2008

To access this journal online:

http://www.birkhauser.ch 